\title{
CONTAMINACIÓN POR COMPUESTOS ORGANOCLORADOS EN ALGUNOS ALIMENTOS PROCEDENTES DE UNA REGIÓN DE MEXICO
}

\author{
Lilia A. Albert* \\ Jaime Rendón-von Osten*
}

\begin{abstract}
ALBERT, L.A. \& RENDÓN.VON OSTEN, J. Contaminación por compuestos organoclorados en algunos alimentos procedentes de una región de México. Rev. Saúde públ., S. Paulo, 22:500-6, 1988.

RESUMEN: Fueran determinados los residuos de plaguicidas organoclorados en huevos y quesos de la población agricola de la Villa de Ahome, Sinaloa, México, mediante cromatografia gas-líquido con detector de captura de eletrones. En los huevos, los compuestos detectados con mayor frecuencia y concentración fueran: epóxido de heptacloro, p, p'-DDT e p, p'-DDE. Simultáneamente fué realizado en la Villa de Ahome un estudio epidemiológico preliminar en la cual se detectó varias manifestaciones patológicas en las personas entrevistadas y en sus familiares.
\end{abstract}

DESCRIPTORES: Insecticidas organoclorados, analisis. Residuos de pesticidas, analisis. Queso, analisis. Huevos, analisis. Contaminación química de alimentos, analisis.

\section{INTRODUCCION}

El hombre, al utilizar plaguicidas sintéticos para diversos fines (agricultura, ganadería, salud pública, etc.) ha contaminado casi todos los sustratos del ambiente biótico y abiótico; esto ha ocurrido a causa de la fácil dispersión de estos compuestos y, principalmente, por la gran persistencia de muchos de ellos en el ambiente. Dicha contaminación de los ecosistemas ha originado problemas muy graves como el de la presencia de residuos de plaguicidas en los alimentos.

Los organoclorados son los plaguicidas que han despertado una mayor preocupación debido a sus efectos indeseables sobre los seres vivos y el medio ambiente. Estos productos son muy insolubles en agua, son solubles en compuestos de baja polaridad, muy estables (su vida media es superior a los diez anos), bioacumulables y, muchas veces, sus productos de degradación son más tóxicos o persistentes que el compuesto original (Brooks $\left.{ }^{6}, 1977\right)$.

Los compuestos organoclorados causan graves efectos toxicológicos, entre ellos, la alteración de la respuesta inmunológica del organismo (Kagan $\left.{ }^{14}, 1985\right)$.

El DDT y sus análogos tienen efectos adversos sobre la corteza suprarrenal y ocasionan manifestaciones antiesteroidales, además de inducir las oxidasas microsomales (Murphy ${ }^{21}$, 1980). Se ha comprobado experimentalmente que varios compuestos organoclorados inducen teratogénesis y mutagénesis (Epstein ${ }^{8}, 1977$; Kaloyanova $\left.{ }^{15}, 1982\right)$.

Debido a las propiedades fisicoquímicas de los plaguicidas organoclorados, el ganado que ha estado expuesto a ellos - ya sea directa o indirectamente - secreta el compuesto original y sus productos de biotransformación junto con la leche; por lo tanto, los derivados lácteos contienen estos productos en elevadas concentraciones, aún después de su procesamiento (Kenaga'", 1972).

Por otra parte, las aves de corral también pueden estar expuestas a los plaguicidas - ya sea por el consumo de alimentos contaminados o por su uso en los gallineros - y eliminan los residuos de dichos compuestos a través del huevo. Por lo general, las concentraciones de residuos de plaguicidas en huevo están directamen. te relacionadas con las concentraciones del plaguicida en el alimento del ave (Foster ${ }^{10}, 1974$; Liska \& Stadelman ${ }^{18}$, 1969).

Sinaloa es uno de los estados de la República Mexicana con mayor producción agrícola, ya que aporta una tercera parte de la exportación agropecuaria del país. Para este estudio se elogió la Villa de Ahome, Sinaloa; esta población se encuentra muy cercana a campos agrícolas que son asperjados contínuamente con plaguicidas; además, el agua para consumo humano se toma de los canales que provienen de los distritos de riego, así como de pozos cercanos a los

\footnotetext{
- Instituto Nacional de Investigaciones sobre Recursos Bióticos. Apdo. Postal 63 (INIREB) - 91000 Xalapa, Veracruz
} - México. 
campos de cultivos que tienen menos de cuatro metros de profundidad.

Los objetivos del estudio fueron: 1) identificar y cuantificar los residuos de plaguicidas organoclorados en queso y huevo de gallina procedentes de la Villa de Ahome, Sinaloa; 2) comparar los resultados con los obtenidos en otros países y, 3) con base en los resultados, sugerir los estudios químicos y epidemiológicos que deban realizarse posteriormente en la zona.

\section{MATERIALES Y METODOS}

La Villa de Ahome en Sinaloa, México, es una comunidad de menos de 15,000 habitantes; por lo tanto, sólo se pudo recolectar y efectuar el análisis en cinco lotes diferentes de huevo y en cuatro de queso, los cuales se obtuvieron en tiendas y casas particulares. Se obtuvieron muestras que provenían exclusivamente de la Villa de Ahome y se aseguró que no se trataba de productos que hubieran procedido de otro sitio.

Después de eliminar el cascarón de los huevos, los lotes se homogeneizaron por separado. Tanto los huevos ya homogeneizados como los quesos, se guardaron en frascos de vidrio color ámbar con tapa de rosca y contratapa de teflón y se conservaron a $-20^{\circ} \mathrm{C}$ hasta su análisis.

En ambos casos, la extracción de los lípidos se realizó con $25 \mathrm{~g}$ de Florisil desactivado al $5 \%$ y $200 \mathrm{ml}$ de una mezcla cloruro de metileno: éter de petróleo, (40:60, v/v) $\left(\mathrm{FDA}^{9}, 1978\right)$.

Los lípidos del queso se purificaron en una columna cromatográfica preparada con $10 \mathrm{~g}$ de alúmina desactivada al $3 \%$ y $20 \mathrm{~g}$ de Florisil activado, la cual se eluyó con $200 \mathrm{ml}$ de una mezcla cloruro de metileno: hexano $(25: 75$, v/v) (Albert et al. ${ }^{4}, 1986$ ).

Los lípidos del huevo se purificaron de la misma manera sólo que la columna se eluyó con $200 \mathrm{ml}$ de una mezcla éter de petróleo: éter etílico $(85: 15, v / v)\left(\right.$ FDA $\left.^{9}, 1978\right)$.
Los análisis cualitativo, cuantitativo y de confirmación se llevaron a cabo de un cromato. grafo gas-líquido con detector de captura de electrones de ${ }^{63} \mathrm{Ni}$ (Varian 3700). Se emplearon dos fases estacionarias: SP-2250 1.5\% / SP-2401 1.95\% sobre Supelcoport 100/120 Supelco, Inc.) y 0V-210 3\% sobre Gas Chrom Q 100/120 (Applied Science Laboratories, Inc.)

\section{RESULTADOS Y DISCUSION}

En la Tabla 1 se presentan los resultados del análisis cualitativo de residuos de compuestos organoclorados en huevo de gallina y en queso.

Como se observa, todas las muestras presentaron residuos de plaguicidas organoclorados 0 de sus productos de biotransformación, los que variaron de uno a tres compuestos por muestra. El p, p'-DDE se identificó en el $100 \%$ de las muestras de huevo y queso, mientras que el epóxido de heptacloro sólo se identificó en el $40 \%$ de las muestras de huevos y el dieldrín en el $25 \%$ de las de quesos. Asimismo, el $80 \%$ de los huevos presentaron p, p'-DDT, el que no se identificó en los quesos.

Por lo anterior, se puede suponer que las aves de corral de esta Villa están expuestas directamente a los plaguicidas organoclorados; no así el ganado lechero, al cual quizás no se le esté aplicando directamente este tipo de sustancias ya que, de lo contrario, el queso presentaría residuos de plaguicidas y no de dieldrín y $\mathrm{p}$, p'-DDE, los cuales son productos de biotransformación del aldrín y del p, p'-DDT, respectivamente.

Las concentraciones de los residuos de estos compuestos en huevo de gallina se presentan en la Tabla 2. Como se puede observar, las muestras 2 y 3 presentaron las mayores concentraciones de DDT equivalente total. Las muestras 3 y 4 presentaron epóxido de heptacloro, el cual es el principal producto de transformación del heptacloro.

TABLA 1

Identificación de residuos de plaguicidas organoclorados em huevo de gallina y queso procedentes de la Villa de Ahome, Sinaloa, Mexico.

\begin{tabular}{|c|c|c|c|c|c|c|c|c|c|c|c|}
\hline \multirow[b]{2}{*}{ Compuesto } & \multicolumn{6}{|c|}{ Huevo } & \multicolumn{5}{|c|}{ Queso } \\
\hline & 1 & 2 & 3 & 4 & 5 & $\%$ & 1 & 2 & 3 & 4 & $\%$ \\
\hline $\begin{array}{l}\text { E.H.* } \\
\text { Dieldrín }\end{array}$ & & & + & + & & 40 & & 1 & & & \\
\hline $\begin{array}{l}\text { Drelarin } \\
\text { p,p' - DDE }\end{array}$ & + & + & $\begin{array}{l}+ \\
+\end{array}$ & + & + & $\begin{array}{r}100 \\
80\end{array}$ & + & $\begin{array}{l}+ \\
+\end{array}$ & + & + & $\begin{array}{r}25 \\
100\end{array}$ \\
\hline
\end{tabular}

* Epóxido de heptacloro 
TABLA 2

Resíduos de compuestos organoclorados en huevos de gallina procedentes de la Villa de Ahome, Sinaloa*

\begin{tabular}{|c|c|c|c|c|c|c|c|}
\hline Compuesto & $\mathbf{M}$ & $\begin{array}{l}\mathrm{U} \\
1\end{array}$ & $\begin{array}{l}\mathrm{E} \\
2\end{array}$ & $\begin{array}{l}\mathbf{S} \\
3\end{array}$ & $\begin{array}{l}T \\
4\end{array}$ & $\begin{array}{l}R \\
5\end{array}$ & $A$ \\
\hline $\begin{array}{l}\text { E.H. }{ }^{* *} \\
\text { p, p' - DDE } \\
\text { p, p' - DDT } \\
\Sigma \text { DDT }\end{array}$ & & $\begin{array}{l}\text { n.d. } \\
0.093 \\
0.024 \\
0.117\end{array}$ & $\begin{array}{l}\text { n.d. } \\
0.382 \\
0.067 \\
0.449\end{array}$ & $\begin{array}{l}0.015 \\
0.125 \\
0.333 \\
0.458\end{array}$ & $\begin{array}{l}0.007 \\
0.129 \\
0.036 \\
0.165\end{array}$ & $\begin{array}{l}\text { n.d. } \\
0.002 \\
\text { n.d. } \\
0.002\end{array}$ & \\
\hline
\end{tabular}

- Concentración em $\mu \mathrm{g} / \mathrm{g}$ (ppm), con base en los lípidos extraŕdos

* Epóxido de heptacloro

n.d. No detectable.

Es importante mencionar que a través del huevo son excretados el $10 \%$ del epóxido de heptacloro, $25 \%$ del $\Sigma D D T$ y fel $35 \%$ del hexaclorobenceno de la ingesta diaria de plaguicidas organoclorados en gallinas (Kan \& Tuinstra ${ }^{16}$, 1976). Por lo anterior, se infiere que las gallinas de la Villa de Ahome se encuentran expuestas de manera crónica a concentraciones considerables de plaguicidas organoclorados y que, sin lugar a dudas, en los tejidos de estas aves se deben estar bioacumulando lentamente estas substancias. Por lo tanto, la población humana, al consumir carne de pollo y huevo puede, a su vez, bioconcentrar dichos residuos, con las consiguientes repercusiones toxicológicas que se manifestarán, sobre todo, a largo plazo.

En la Tabla 3 se presentan las concentraciones de compuestos organoclorados determinadas en los quesos procedentes de la Villa de Ahome. Se aprecia que todas las muestras tuvieron $\mathrm{p}, \mathrm{p}$ '-DDE, el cual tuvo una concentración media de $0.034 \mu \mathrm{g} / \mathrm{g}(\mathrm{ppm})$ y que sólo una muestra presentó dos compuestos: dieldrín y $\mathrm{p}$, p'-DDE.

Con base en estos resultados, se puede postular que los plaguicidas organoclorados han dejado de usarse ampliamente en esta zona, ya que la contaminación de los quesos con estas sustancias es consecuencia de la concentración de éstas a través de la red trófica y su bioacumulación.

\section{TABLA 3}

Residuos de compuestos organoclorados en queso procedente de la Villa de Ahome, Sinaloa*

\begin{tabular}{|c|c|c|c|c|c|c|}
\hline Compuesto & $\begin{array}{r}M \\
1\end{array}$ & $\mathrm{U}$ & $\begin{array}{c}\mathrm{E} \\
2\end{array}$ & $\mathrm{~S}$ & $\mathrm{~T}_{3} \mathrm{R}$ & $\begin{array}{l}\mathrm{A} \\
4\end{array}$ \\
\hline $\begin{array}{l}\text { Dieldrín } \\
\mathrm{p}, \mathrm{p}^{\prime}-\mathrm{DDE}\end{array}$ & $\begin{array}{l}\text { n.d. } \\
0.055\end{array}$ & & $\begin{array}{l}0.114 \\
0.015\end{array}$ & & $\begin{array}{l}\text { n.d. } \\
0.015\end{array}$ & $\begin{array}{l}\text { n.d. } \\
0.051\end{array}$ \\
\hline
\end{tabular}

Concentración en $\mu \mathrm{g} / \mathrm{g}(\mathrm{ppm})$, con base a lípidos extraídos

n.d. No detectable
Lo anterior se confirma en la Tabla 4, en la que se comparan estos resultados con las concentraciones de residuos de plaguicidas organoclorados determinadas en quesos procedentes de Culiacán, Sinaloa (Albert \& Reyes ${ }^{3}, 1978$ ), ciudad que se encuentra aproximadamente a $200 \mathrm{~km}$ de la Villa de Ahome, en el mismo Estado.

TABLA 4

Comparación de los intervalos de concentración de residuos de compuestos organoclorados em queso*

\begin{tabular}{lcc}
\hline Compuesto & $\begin{array}{c}\text { Culiacán, } \\
\text { Sinaloa } \\
(1978)^{4}\end{array}$ & $\begin{array}{c}\text { Villa de Ahome } \\
\text { Sinaloa } \\
(1986)\end{array}$ \\
\hline$\alpha$ HCH & $0.056-0.139$ & - \\
Dieldrín & - & n.d. -0.114 \\
p,p'-DDE & $0.200-1.170$ & $0.015-0.051$ \\
p,p' - DDT & $0.200-0.700$ & - \\
\hline
\end{tabular}

* Concentraciones em $\mu \mathrm{g} / \mathrm{g}$ (ppm), con base a lípidos extraídos.

n.d. No detectable.

Así, se observa que en los quesos procedentes de Culiacán se encontraban presentes los compuestos p, p'-DDT, p, p'-DDE y $\alpha \mathrm{HCH}$ en la época de dicho estudio, y que la concentración de $p, p$ '-DDE era de 10 a 20 veces mayor que la actual en la Villa de Ahome; por lo tanto, se puede suponer que el $p$, p'-DDT utilizado en aquel entonces se ha degradado para dar $p$, p'-DDE, el cual se presenta claramente en los resultados obtenidos en este estudio. Sin embargo, no se debe desechar la posibilidad de que en algún momento dado se hayan usado en la región otros plaguicidas tales como el aldrín.

En la Tabla 5 se presenta la comparación de los resultados obtenidos en huevos de gallina de España (Serrano et al. ${ }^{23}$, 1979) e Irán (Hashemy \& Mosstofian ${ }^{11}$, 1979) y los del presente estudio.

Como se observa, las concentraciones promedio de $\mathrm{p}$, p'-DDE fueron mayores en el presente estudio, con relación a España e Irán en 
TABLA 5

Comparación de las concentraciones medias de residuos de compuestos organoclorados em huevo de gallina de tres paises*

\begin{tabular}{lccc}
\hline Compuesto & $\begin{array}{c}\text { México } \\
1986\end{array}$ & $\begin{array}{c}\text { España*** } \\
1979\end{array}$ & $\begin{array}{c}\text { Irán**** } \\
1979\end{array}$ \\
\hline$\gamma$ HCH & & 0.100 & \\
$\Sigma$ HCH & & 0.520 & \\
Heptacloro/E.H."**** & 0.010 & 0.010 & 0.023 \\
Aldrín/dieldrín & & 0.080 & 0.031 \\
p,p'-DDE & 0.146 & 0.030 & 0.087 \\
p,p'-DDD & & 0.020 & 0.012 \\
p,p'-DDT & 0.114 & 0.170 & 0.140 \\
$\Sigma$ DDT & 0.260 & 0.220 & 0.239 \\
Endrín & & & 0.017 \\
\hline
\end{tabular}

* Concentraciones en $\mu \mathrm{g} / \mathrm{g}$ (ppm); con base lípidos extraídos

** Serrano et al ${ }^{23}$ (1979)

*** Hashemy - Tunkabony e Masstofian ${ }^{11}$ (1979)

**** Epóxido de heptacloro.

aproximadamente un 40 y $80 \%$, respectivamente.

Las concentraciones promedio de p, p'-DDT para las muestras de España e Irán son muy similares entre sí mientras que las obtenidas en los productos de la Villa de Ahome fueron ligeramente menores; sin embargo; la concentración de $\Sigma D D T$ fue mayor en México en un 44 y $40 \%$ respectivamente en relación con dichos países, aún cuando en estos últimos se describió la presencia de p, p'-DDD en el huevo de gallina.

Esto hace suponer que, en aquellos países, las aves de corral consumieron alimento vegetal que estaba contaminado con residuos de plaguicidas organoclorados, ya que las plantas biotransforman el $\mathrm{p}, \mathrm{p}$ '-DDT principalmente a $\mathrm{p}$, p'-DDD, y no a p, p'-DDE, como ocurre generalmente en las aves y mamíferos (Matsumu$\left.\mathrm{ra}^{19}, 1975\right)$. Por lo anterior, se sostiene la hipotesis de que las aves de corral de la Villa de Ahome están directamente expuestas a los plaguicidas organoclorados.

En la Tabla 6 se presenta la comparación de las concentraciones promedio de residuos de compuestos organoclorados en quesos de la Villa de Ahome, México e Italia (Cerruti et al.?, 1975). Se puede observar que en Italia existían en la fecha de ese estudio problemas de contaminación por residuos de plaguicidas organoclorados en quesos, ya que en ese país se determinaron cinco compuestos diferentes y en la Villa de Ahome, México sólo dos. Las concentraciones de $p$, p'-DDE fueron muy similares en los dos estudios. En los quesos de México se halló además, dieldrín, el cual se extremadamente tóxico a corto y a largo plazo (Kagan ${ }^{14}$, 1985).

Es importante hacer notar que los resultados de Italia corresponden a 1975, cuando las restricciones en el uso de plaguicidas perjudiciales apenas se estaban poniendo en práctica, mientras que en México, aún no existe un control sobre los residuos de plaguicidas en alimentos.

Además, es importante mencionar que de diez paises en los que se analizaron plaguicidas organoclorados en alimentos tales como leche y pescado, las concentraciones de DDT y hexaclorociclohexano fueron mayores en México, China e India que en países en los cuales la legislación sobre el uso de estos agrotóxicos se ejerce eficientemente (MARC $\left.{ }^{20}, 1987\right)$.

\section{TABLA 6}

Comparación de concentraciones promedio de residuos de compuestos organoclorados en quesos de Mexico e Italia*

\begin{tabular}{lcc}
\hline Compuesto & $\begin{array}{c}\text { México } \\
1986\end{array}$ & $\begin{array}{c}\text { Italia** } \\
1975\end{array}$ \\
\hline$\gamma$ HCH & & 0.036 \\
$\Sigma$ HCH & & 0.080 \\
Dieldrín & 0.114 & \\
p, p' - DDE & 0.033 & 0.038 \\
p, p' - DDD & & 0.046 \\
p,p' - DDT & & 0.124 \\
\hline
\end{tabular}

* Concentraciones em $\mu_{g} / g$ (ppm); con base lípidos extraídos

** Cerruti et al ${ }^{7}$ (1975)

Al comparar los resultados de este estudio con los límites permisibles de residuos de plaguicidas organoclorados en alimentos propuestos por la FAO/OMS y algunos países desarrollados (Tabla 7), se observa que las concentraciones determinadas en México son ligeramente inferiores a los límites recomendados por la FAO/OMS ${ }^{12}$ (1986) y los Estados Unidos de América $\left(\mathrm{FDA}^{9}, 1978\right)$, mientras que la Unión Soviética (MHW $\left.{ }^{22}, 1974\right)$ no permite la presencia de estos residuos en los alimentos.

Sin embargo, las concentraciones determinadas para la ingestión de estos residuos en huevo y queso de la Villa de Ahome son mayores que la Ingesta Diaria Admisible (IDA).

Si se recuerda que en general la alimentación. de la población mexicana se basa en productos agrícolas, y que un mexicano puede consumir dos huevos y un poco más de $100 \mathrm{~g}$ de queso diariamente, se puede deducir que las concentraciones que generalmente ingiere la población de la Villa de Ahome sobrepasan la IDA. 
TABLA 7

Limites maximos permisibles para residuos de plaguicidas organoclorados en alimentos e ingesta diaria admisible*

\begin{tabular}{lcccc}
\hline Compuesto & FAO/OMS** & EUA*** & URSS**** & IDA***** \\
\hline Aldrín/ & 0.15 & 0.03 & N.P. & 0.0001 \\
Dieldrín & 0.15 & 0.03 & N.P. & 0.0050 \\
Heptacloro/ & 1.25 & 1.50 & N.P. & 0.0050 \\
Epóx. Heptacloro & D DDT & &
\end{tabular}

IDA Ingestión diaria admisible $(\mathrm{mg} / \mathrm{kg})$

NP No permitido.

* Concentraciones em $\mu \mathrm{g} / \mathrm{g}$ (ppm); con base lípidos extraídos

** FAO/OMS ${ }^{13}(1986)$

*** FDA $^{1}(1973)$

**** MHW22 (1974)

***** FAO/OMS 12 (1979)

En la Tabla 8 se presentan los resultados de dichos cálculos; se observa que los residuos ingeridos de $p, p^{\prime}$-DDE y $p, p^{\prime}-D D T$ en huevo son 43 y 33 veces mayores que la IDA. En queso, los resultados son aún mayores ya que los residuos de dieldrín exceden más de 500 veces la IDA.

Asimismo, los resultados de las concentraciones ingeridas de $p, p^{\prime}-D D E$ en queso y epóxido de heptacloro en huevo son un poco más de dos veces mayores que la IDA.

Cabe señalar que muchas veces los límites prácticos para residuos de plaguicidas organoclorados en alimentos, que han sido propuestos por los países y los diversos organismos internacionales, no toman en consideración los efectos a largo plazo y las interacciones de dos o más sustancias químicas dentro del organismo tales como los efectos aditivo, sinérgico y de potenciación; tampoco se toma en cuenta el estado nutricional, a pesar de que, en general, una dieta baja en proteinas aumenta la toxicidad de un plaguicida (Almeida ${ }^{5}, 1982$ ). Al respecto, cabe recordar que en los países latinoamericanos, incluyendo a México, las condiciones de salud y nutrición están muy por debajo de las consideradas normales.

A la par del estudio químico aquí descrito, se realizó una encuesta epidemiológica preliminar con 41 personas de la Villa de Ahome. En ella se encontró que existe un elevado número de casos de alcoholismo y de tabaquismo, que la nutrición y la higiene son deficientes y que la eliminación de desechos es inadecuada. Se registraron también $39 \%$ de casos de diabetes mellitus, $19 \%$ de manifestaciones alérgicaś, $10 \%$ de casos de cáncer, $24 \%$ de casos de familiares con cáncer y 14 abortos espontáneos entre las 27 mujeres entrevistadas.

En la Villa de Ahome no sólo existe el problema de los plaguicidas organoclorados sino también en de otros tipos de plaguicidas ya que, por ejemplo, en 1985 se emplearon, entre otros, 19 tipos diferentes de insecticidas, 19 de herbicidas y 9 de fungicidas, lo cual sumo $64,849 \mathrm{~kg}$ de plaguicidas*. Estas cantidades representan sólo el $20 \%$ del total, ya que el $80 \%$ del área cultivada en la Villa de Ahome pertenece a pequeños propietarios, los cuales están fuera del control estadístico de las autoridades.

\section{TABLA ?}

Concentración promedio de ingesta diaria en huevo y queso de la Villa de Ahome, Sinaloa.

\begin{tabular}{lcccr}
\hline & $\begin{array}{c}\text { Alimento } \\
\text { Diário }\end{array}$ & Compuesto & $\begin{array}{c}\text { Ingesta } \\
\text { Calculada }\end{array}$ & Ingesta/IDA \\
\hline Huevo (2) & $100 \mathrm{~g}$ & p,p' - DDE & 14.0 & 43.07 \\
& & p,p' - DDT & 11.0 & 33.84 \\
Queso & & E.H.** & 1.0 & 3.07 \\
& $100 \mathrm{~g}$ & p,p' - DDE & 0.9 & 2.76 \\
\hline
\end{tabular}

* Calculada para una persona de $65 \mathrm{~kg}(\mathrm{mg} / \mathrm{kg})$

** Epóxido de heptacloro.

A. Piffa Paez, Comunicación personal, SARH. 
Se sabe que las aplicaciones de agrotóxicos sobre hortalizas en el Valle de Culiacán son extremadamente altas en relación con otros cultivos y otras regiones del país (Wright ${ }^{24}, 1984$ ). Los datos sobre residuos de plaguicidas organoclorados en aguas del sistema de drenaje del Distrito de Riego N: 10 del Estado de Sinaloa demuestran que en él las concentraciones de ciertos plaguicidas, tales como lindano, aldrín, heptacloro, clordano, eldrín y p, $\dot{p}$ '-DDT, excedieron los límites establecidos por la Agencia de Protección Ambiental de los EUA (US-EPA) (Albert \& Armienta ${ }^{2}$, 1977).

Por lo anterior, se recomienda hacer un seguimiento en estudios químicos de plaguicidas en la Villa de Ahome tomando en consideración los parámetros ecológicos correspondientes. Estos análisis deben ser, preferentemente, en agua y aire ya que, según pláticas con algunos habitantes, se puede postular que estos son los sustratos ambientales más contaminados en dicha Villa; además, se deben hacer determinaciones de otros tipos de plaguicidas, tales como organofosforados y carbámicos.

Por otra parte, es necesario realizar en la zona una campana de educación ambiental en todos los niveles posibles acerca del peligro potencial que representa el mal uso de los plaguicidas.

Lo más importante es la necesidad de que en México se lleve a cabo un control eficiente del uso de agrotóxicos; en especial de los organoclorados, para evitar que sea indiscriminado ya que, en los países desarrollados, el registro de estos productos ya ha sido cancelado, restringido o suspendido debido a los efectos adversos como cáncer, mutaciones y teratogénesis que originan en animales de experimentación, y a las alteraciones que causan en especies no nocivas.

ALBERT, L.A. \& RENDÓN-VON OSTEN, J. Contaminação por compostos organoclorados em alguns alimentos procedentes de uma região do México. Rev. Saúde públ., S. Paulo, 22:500-6, 1988.

RESUMO: Foram determinados os resíduos de praguicidas organoclorados em ovos e queijos da população agrícola da Villa de Ahome, Sinaloa, México, mediante cromatografia gás-líquido com detector de captura de elétrons. Nos ovos, os compostos detectados com maior frequência e concentraçào foram: epóxido de heptacloro, $\mathbf{p}, \mathbf{p}^{\prime}$-DDT e $\mathbf{p}, \mathbf{p}^{\prime}$-DDE; nos queijos os contaminantes mais frequientes foram: dieldrín e p, p'-DDE. Simultaneamente foi realizado na Villa de Ahome um estudo epidemiológico preliminar no qual se detectou várias manifestaçőes patológicas nas pessoas entrevistadas e em seus familiares.

UNITERMOS: Inseticidas organoclorados, análise. Resíduos de pesticidas, análise. Queijo, análise. Ovos, análise. Contaminação quimica de alimentos, análise.

ALBERT, L.A. \& RENDÓN-VON OSTEN, J. [Contamination by organochlorine compounds in some foodstriffs from Region of Mexico]. Rev. Saúde públ., S. Paulo, 22: $\quad, 1988$.

ABSTRACT: Organochlorine pesticide residues were determined in hen's eggs and cheese samples from the agricultural town Villa de Ahome, Sinaloa, México, using gas-liquid chromatography with electron capture detection. In eggs, the compounds identified most frequently and in greatest concentrations were heptachlor epoxide, $p, p^{\prime}-D D T$ and $p, p^{\prime}-D D E$, while in cheese the most frequent contaminants were, p, p'-DDE and dieldrin. Simultaneously, by means of a preliminary epidemiological survey several pathologies prevalent in the area were detected.

UNITERMS: Insecticides, organochlorine, analysis. Pesticide residues, analysis. Cheese, analysis. Eggs, analysis. Food contamination, chemical, analysis.

\section{REFERENCIAS BIBLIOGRAFICAS}

1. ADMINISTRATIVE Guidelines Manual. Washington, D.C., U.S. Department of Health, Education and Welfare, Food and Drug Administration, 1973.

2. ALBERT, L.A. \& ARMIENTA, V. Contaminación por plaguicidas organoclorados en un sistema de drenaje agrícola en el Estado de Sinaloa. Proteccion de la Calidad del Agua, 1(3):5-17, 1977.

3. ALBERT, L.A. \& REYES, R. Contaminación de algunos quesos mexicanos por plaguicidas organoclorados. Rev. Soc. Quim. Mex., 22:65-72, 1978.
4. ALBERT, L.A.; RENDÓN-VON OSTEN, J.; ALPUCHE, L. Método práctico de purificación de lipidos para el análisis de plaguicidas organoclorados en quesos. Rev. Soc. Quím. Mex., 30:258, 1986.

5. ALMEIDA, W. Fundamentos toxicológicos de los plaguicidas. In: Plaguicidas: Salud y Ambiente, San Cristobal de las Casas, Chiapas, México, 1982; Memorias del Taller. Xalapa, México, Centro Panamericano de Ecología Humana y Salud de la OPS/INIREB, 1982. p.62-74. 
6. BROOKS, G.T. Chlorinated insecticides: restrospect and prospect. In: Plimmer, J.R. Pesticide chemistry in the $20^{\text {th }}$ century. Washington, D.C., 1977, p.1-20. (ACS Symposium Series 37).

7. CERRUTI, G.; ZAPPAVIGNA, R.; CEROSA, S. Residui di antiparassitari cloro - e fosfo - organici in latte e derivatti. Il Latte, 3(5):161-75, 1975.

8. EPSTEIN, S.S. The carcinogenicity of organochlorine pesticides. In: Hiatt, H.H.; Watson, J.D.; Winsten, J.A. Origens of human cancer. Cold Spring Harbor, N.Y., Cold Spring Harbor Laboratory, 1977. p.24365.

9. FOOD DRUG ADMINISTRATION. Pesticide analytical manual: Section chlorinated 2.21 (A). Chlorina. ted organic pesticides in fatty foods. Washington, D.C., 1978. v. 1.

10. FOSTER, T.S. Physiological and biological effects of pesticide residues in poultry. Residue Rev., 29:13-37, 1974.

11. HASHEMY-TUNKABONY, S.E. \& MOSSTOFIAN, B. Chlorinated pesticide residues in chicken eggs. Poult. Sci., 58:1432-4, 1979.

12. JOINT FAO/WHO FOOD STANDARDS PROGRAMME. Codex Alimentarius Commision, Rome, 1979. 13th Session. Rome, 1979.

13. JOINT FAO/WHO FOOD STANDARDS PROGRAMME. Codex Alimentarius Commision. Guide to Codex Recommendations concerning pesticide residues. Part 2: Maximun limits for pesticide residues. Rome, 1986.

14. KAGAN, Y.S. Principles of pesticide toxicology. Moscow, International Projects. UNEP/IRPTC, 1985.

15. KALOYANOVA, F. Organochlorine pesticides. In: Kaloyanova, F. \& Tarkowski, S. Toxicology of pesticides: health aspects of chemical safety. Copenha- gen, World Health Organization, 1982. p.123-32. (Interim Document, 9).

16. KAN, C.A. \& TUINSTRA, L.G.M.T. Accumulation and excretion of certain organochlorine insecticides in broiler breeder hens. J. Agr. Food Chem., 24:775. $8,1976$.

17. KENAGA, E.E. Factors related to bioconcentration of pesticides. In: Matsumura, F.; Mallory, G.; Misato, T. Environmental toxicology of pesticides. New York, Academic Press, 1972. p.193-228.

18. LISKA, B.J. \& STADELMAN, W.J. Accelerated removal of pesticides from domestic animals. Residue Rev., 29:51-9, 1969.

19. MATSUMURA, F. Toxicology of pesticides. New York, Plenum Press, 1975. p.50-60.

20. MONITORING AND ASSESSMENT RESEARCH CENTRE (MARC). Clean air, pure water and safe food and healthy world. Sentinel Hith Environ. Int., 4:5-6, 1987.

21. MURPHY, S.D. Pesticides. In: Doull, J.; Klaasen, C.D.; Amdur, M.O. Toxicology, the basic science of poisons. New York, MacMillan Publ., 1980. p.357408.

22. NATIONAL pesticide residue tolerances. Ottawa, Canada, Bureau of Chemical Safety. Ministry of Health and Welfare, 1974.

23. SERRANO, J.M.; GARCÍA, A.; SANTIAGO, D.; INFANTE, F. Residuos de pesticidas organoclorados en yema de huevo. Arch. Zootec., 28:137-42, 1979.

24. WRIGHT, A. Una investigación del uso de plaguicidas en el Valle de Culiacán, Sinaloa, México. Sacramento, California State University, 1984.

Recebido para publicação em 26/4/1988 Aprovado para publicaçāo em 20/6/1988 